\title{
CORONAL HOLES AND THE SOLAR WIND
}

\author{
EGII, LEFR \\ Institute of Theoretical Astrophysics, University of Oslo \\ P O Box 109.9, Blindern, N-0.316 Oslo, Norway
}

\section{Historical background}

Around the turn of the century Professor Kristian Birkeland at the University of Kristiania (Oslo) carried out laboratory studies of the emission of charged particles from the Sun and their interaction with comets and magnetized planets. He found that cathode rays emitted by a magnetized sphere are bent toward the equator along trajectories similar to the structures observed in the solar corona during the 1901 eclipse. He also concluded that comet tails are formed by charged particles from the Sun interacting with dust and gas around the comet.

Birkeland carricd out extensive observing programs of the aurora, geomagnetic storms, and the zodiacal light. During a 9 months campaign from mid 1902 he observed recurrent geomagnetic storms from stations in Northern Norway, at Nova ja Semlja, Iceland, and Spitsbergen, and correlated the occurence and periodicity of magnetic storms with solar activity. He found that the period of recurrent geomagnetic storms was generally larger than the period of "near equator" solar phenomena. He also found that "Very often large maxima of storminess are not accompanied by any sun-spots at all". (Birkeland 1913, p. $524)$.

Birkeland's studies of the emission of particles from the Sun and their interaction with bodies in interplanctary space led him to write: "It seems to be a natural consequence of our points of view that the whole of space is filled with electrons and flying ions of all kinds. We liave assumed that each stellar system in evolutions throws off electric corpuscules into space. It does not seem unreasonable therefore to think that a greater part of the matcrial masses in the universe is found, not in the solar systems or nebulae, but in "empty" space", (Birkeland, 1913; p. 720).

\section{Parker's solar wind model}

Birkeland's work was largely forgotten, and it was the studies of comet tails in Germany that formed the basis for Parker's theoretical studies of the solar wind. Parker (1958) described the expanding, fully ionized coronal hydrogen plasma as a fluid. He argued that the solution of the conservation equations consistent with a large coronal pressure and a low asymptotic pressure (balancing the interstellar pressure) is a subsonic - supersonic solution with a shock far from the Sun. Parker's solution of the equations for steady flow seems to describe the basic physics of the dynamics of the solar wind, but the flow speed near the orbit of Earth is much lower that the values observed in quasi-steady high speed solar wind streams. IIence, the energy balance in the flow is not properly described in the 
model.

In the 1960's it was shown that recurrent geomagnetic storms are caused by quasisteady high speed solar wind streams. Some ten years later it was established that large coronal holes are the source regions of these high speed streams. During the declining phase of the sun-spot cycle the polar regions develop into large coronal holes, and when these extend to lower solar latitudes the high speed streams are observed in the ecliptic (e.g. Hundhausen, 1977).

\section{Mass- and energy balance in the solar wind}

Acceleration of the quasi-steady high speed solar wind to flow speeds of $700-800 \mathrm{~km} / \mathrm{s}$ require an energy source in addition to thermal energy being conducted outward from the solar corona and transferred to the flow by the pressure gradient force. Leer and Holzer (1980) showed that energy must be added to the supersonic region of the flow to increase the asymptotic flow speed; energy addition close to the Sun leads to a larger density scale height in the corona and a larger mass flux in the solar wind. It has been demonstrated that Alfven waves of solar origin deposit most of their energy flux in the supersonic region of the flow, and can indeed accelerate the flow to $700-800 \mathrm{~km} / \mathrm{s}$.

In most solar wind models the flow is assumed to consist of electrons and protons. The proton (and electron) flux is such models is a sensitive function of coronal temperature: $A$ variation of the coronal temperature from 1 to 2 million degrees is consistent with a variation of the proton flux with a factor 100 or more. The observed variation of the proton flux is consistent with a so small temperature variation in the coronal source regions that we must consider regulation mechanisms for the proton flux. Here, we will mention one possible regulation mechanism, namely alpha-particles in the solar corona.

In situ observations show that the flux of alpha-particles in the solar wind varies from 0 to 0.3 of the proton flux, and for quasi-steady high speed streams it is around 0.05 . An alpha-particle flux of 0.05 of the proton flux is not inconsistent with a large alpha-particle abundance in the solar corona. The transport of alpha-particles into the corona from the lower solar atmosphere is due to the thermal force in the transition region. In a steady state, where the thermal force on the alpha particles is balanced by the pressure gradient force, we have an alpha-particle dominated corona, but if the thermal force is balanced by the collisional drag a corona with a fraction of alpha-particles to protons of $0.2-0.4$ is formed (cf. Ilansteen et al., 1991). Such a consentration of alpha-particles in the corona, which can not be excluded by present observations, will act as a regulator of the solar wind proton flux (cf. Leer and Holzer, 1990).

\section{References}

Birkeland,K.: 1913, The Norwegian Aurora Polaris Expedition 1902-190.3 vol.1, sec.2, Aschehoug, Kristiania.

Hansteen,V., Holzer, T.E., and Leer, E.: 1991, in preparation.

Hundhausen, A..J.: 1977, in Coronal Holes and High Speed Wind Streams .J.B. Zirker, Ed., Colorado Ass. Univ. Press.

Leer, E. and Holzer, T.E.: 1980, J. Geophys. Res. 85, 4681.

Leer, E. and IIolzer, T.E.: 1990, Annales Geophysicae 9, 196.

Parker, E.N.:195R. Astrophys. J. 128, 664. 